\title{
Down syndrome
}

INSERM

\section{Source}

INSERM. (1999). Orphanet: an online rare disease and orphan drug data base. Down syndrome. ORPHA:870

Down syndrome is a chromosomal abnormality caused by the presence of a third (partial or total) copy of chromosome 21 and that is characterized by variable intellectual disability, muscular hypotonia, and joint laxity, often associated with a characteristic facial dysmorphism and various anomalies such as cardiac, gastrointestinal, or endocrine defects. 